\title{
A ureteral stricture disease score and classification system: correlation with upper urinary tract reconstructive surgery complexity
}

\author{
Weijie Zhu ${ }^{1 \#}$, Zhenpeng Zhu ${ }^{1 \#}$, Zhihua Li $^{1 \#}$, Xinfei Li ${ }^{1}$, Jianye Zhang ${ }^{1}$, Yangyang Xu' ${ }^{1}$ Xiang Wang ${ }^{1}$, \\ Peng Zhang, ${ }^{2}$, Bingwei Huang ${ }^{2}$, Chen Huang ${ }^{3}$, Dengxiang Zhang ${ }^{3}$, Hongjian Zhu ${ }^{3}$, Xuesong Li ${ }^{1}, J^{1 a n}$ Lin $^{1}$, \\ Liqun Zhou ${ }^{1}$
}

${ }^{1}$ Department of Urology, Peking University First Hospital, Institute of Urology, Peking University, National Urological Cancer Centre, Beijing, China; ${ }^{2}$ Department of Urology, Emergency General Hospital, Beijing, China; ${ }^{3}$ Department of Urology, Beijing Jiangong Hospital, Beijing, China

Contributions: (I) Conception and design: W Zhu, H Zhu, X Li; (II) Administrative support: J Lin, L Zhou; (III) Provision of study materials or patients: X Li, J Zhang, Y Xv, C Huang, D Zhang; (IV) Collection and assembly of data: X Wang, P Zhang, B Huang; (V) Data analysis and interpretation: Z Zhu, Z Li; (VI) Manuscript writing: All authors; (VII) Final approval of manuscript: All authors.

\#These authors contributed equally to this work.

Correspondence to: Hongjian Zhu. Department of Urology, Beijing Jiangong Hospital, No. 6 Rufuli St, Xicheng District, Beijing 100034, China. Email: hjzhu99@sina.com; Xuesong Li. Department of Urology, Peking University First Hospital, Institute of Urology, Peking University, National Urological Cancer Centre, Beijing, China. Email: pineneedle@sina.com.

Background: To develop an original and standardized ureteral stricture disease (USD) score and classification system for quantifying ureter stricture characteristics, assessing complexity of the minimally invasive upper urinary tract reconstructive (UUTR) surgical procedure, formulating preoperative plans, and offering objective comparisons of surgical techniques between different institutions and surgeons.

Methods: We retrospectively reviewed a test set of 64 patients and a validation set of 170 patients who underwent minimally invasive UUTR surgery from January 2018 to January 2021. Three factors were selected to be included in the USD score and classification system: (I) stricture etiology (E, 1-2 points); (II) stricture segment (S, 0-3 points); and (III) length of stricture (L, 1-5 points). The UUTR surgery involves low-complex surgeries (cystoscopy with ureteral dilation and stent placement, ureteropyeloplasty, end to end repair, ureteral reimplantation) and high-complex surgeries (onlay repair (buccal mucosae, lingual mucosae, appendix mucosae), Boari flap repair and ileal ureter replacement). Estimated blood loss and operative time were used as surrogate indicators of surgical complexity.

Results: The interrater reliability of the USD score and classification system was 0.908. A linear relationship between the USD score and estimated blood loss was observed ( $r s=0.676, \mathrm{P}<0.001$ ). The USD score was also correlated with operative time ( $\mathrm{rs}=0.638, \mathrm{P}<0.001)$. A significant difference in USD scores was found between the high and low complexity surgery groups (4 vs. 7, $\mathrm{P}<0.001)$. Variability of UUTR surgery is based on USD classification system, but with regularity to conform to.

Conclusions: The USD score and classification system is a concise, easily applicable, and validated scale to delineate the clinically significant features of ureter stricture that correlate with the complexity of the UUTR surgical procedure. The use of this score and classification system can facilitate preoperative plan and comparison of USD treatments in clinical practice and urological literature. Research with large sample is needed to further examine and modify the use of the system.

Keywords: Ureter stricture disease (USD); upper urinary tract reconstruction (UUTR); score system; classification system; minimally invasive surgery

$\wedge$ ORCID: 0000-0003-2699-8377. 
Submitted Jun 30, 2021. Accepted for publication Sep 02, 2021.

doi: $10.21037 /$ tau-21-575

View this article at: https://dx.doi.org/10.21037/tau-21-575

\section{Introduction}

Classification systems facilitate the organization of complicated processes into logical units. The main goal of the medical classification system is to aid in clinical communication, to predict clinical outcomes, and promote research on a specific disease process. In urological surgery, several score and classification systems have been proven to be successful, including renal mass complexity classification (1), prostate cancer score systems to predict postoperative outcomes (2), and urethral stricture score related to anterior urethroplasty complexity and recurrence (3). To the best of our knowledge, there is no scoring and classification system for ureter stricture to assist in qualitative description, guide clinical decisions, or to reliably compare surgical procedures among centers and surgeons for clinical trials and research.

Ureteral stricture disease (USD) is characterized by ureteral narrowing leading to functional obstruction. As urine excretion is restricted, urine stagnates in the renal pelvis and ureter. If not treated, this condition of obstructed ureter may lead to dilatation of the upper urinary tract, renal pain, or may even cause renal failure $(4,5)$. The management of USD remains a huge challenge for urologists who faced notably different conditions depending on the stricture etiology, segment, and length (6).

The purpose of this research is to develop a score and classification system for USD that can assess the complexity of the upper urinary tract reconstructive (UUTR) surgical procedure and provide a method to promote surgical decision-making, while also offering objective comparisons of surgical techniques between different institutions and surgeons for clinical trials and research. We present the following article in accordance with the STROBE reporting checklist (available at https:// dx.doi.org/10.21037/tau-21-575).

\section{Methods}

\section{Patients}

We retrospectively reviewed a test set of 64 patients and a validation set of 170 patients who underwent minimally invasive UUTR surgery from January 2018 to January
2021, after we excluded patients for which there was incomplete data on the stricture characteristics or surgery type. The clinical demographic data for patients as well as radiological findings were collected from our RECUTTER database (Reconstruction of Urinary Tract: Technology, Epidemiology and Result, http://pkufh.yorktal.com), and data concerning patients' strictures and surgical treatment were analyzed in the test and validation set.

All procedures performed in this study were in accordance with the Declaration of Helsinki (as revised in 2013) and approved by the Ethics Committee of Peking University First Hospital (approval number: 2020-283). Because of the retrospective nature of the research, the requirement for informed consent was waived.

\section{Proposal of USD score and classification system}

In the test group of 64 patients, we compared the basic information of patients (such as gender, age, BMI, stricture etiology, stricture segment, length of stricture) with surgical information (such as surgical method, operation time, estimated blood loss, postoperative complications, and length of hospital stay). Through correlation analysis of these data, it was found that the stricture etiology, stricture segment and length of stricture were moderately or highly correlated with surgical method, operation time and estimated blood loss. Therefore, the USD score and classification system were based on the primary characteristics of the ureter stricture, namely stricture etiology (E, 1-2 points), stricture segment ( $\mathrm{S}, 0-3$ points), and stricture length (L, 1-5 points), which were tallied to provide a total score of 2 to 10 points (Table 1). Stricture etiology was determined by patient history, and stricture segment and length were measured by perioperative radiological images and later verified at surgery.

And two senior urological surgeons and one junior urological surgeon scored and classified the ureteral stricture by means of perioperative radiological images and operative video data. The three raters were blinded to each other's scores and did not exchange the information about the patients. The data analyst was blinded to the identity the source of all three raters' scores. The interrater reliability of USD score and classification system was 
Table 1 Description of the USD score and classification system

\begin{tabular}{lll}
\hline Classification & Score (points) & Description \\
\hline E-stricture etiology & 1 & Idiopathic \\
E1 & 2 & Acquired (iatrogenic, traumatic, inflammatory, failure of previous surgery) \\
E2 & & \\
S-stricture segment & 0 & Distal ureter segment \\
S0 & 1 & UPJ \\
S1 & 2 & Proximal or middle ureter segment \\
S2 & 3 & Multiple ureter segments \\
S3 & & \\
L-stricture length & 1 & $\leq 2 \mathrm{~cm}$ \\
L1 & 3 & $>2 \&<5 \mathrm{~cm}$ \\
L3 & 5 & $\geq 5 \mathrm{~cm}$ \\
L5 & & \\
\hline
\end{tabular}

USD, ureter stricture disease; UPJ, ureteropelvic junction.

studied.

\section{Surgical technique}

According to the senior surgeon's experience with over 1,000 UUTR surgical procedures, cystoscopy with ureteral dilation and stent placement, ureteropyeloplasty, end-toend repair, and ureteral reimplantation were categorized as low-complex surgeries. Onlay repair (buccal mucosae, lingual mucosae, and appendix mucosae), Boari flap repair, and ileal ureter replacement were categorized as highcomplex surgeries. Estimated blood loss and operative time were used as surrogate indicators of surgical complexity.

\section{Statistical analysis}

Data analysis was performed with SPSS software version 23.0. Descriptive data are presented as percentages and frequency. The $t$-test was used to analyze continuous variables, and the chi-square and Fisher exact tests were used to assess categorical data. The inter-observer reliability test was used in the test set. The relationship between USD score and surgical complexity was determined by the Mann-Whitney $U$-test for the median score in low and high complexity procedures. The Spearman rank correlation was used for measuring statistical dependence between USD score and estimated blood loss (EBL) or operative time.

\section{Results}

Patients' demographics for the test and validation cohorts are separately detailed in Tables 2,3. Among the test cohorts, the USD score and classification system were found to have an interrater reliability of $0.908(\mathrm{P}<0.001)$ by means of Kendall's coefficient of concordance, indicating substantial agreement among the reviewers. In this study, the Spearman correlation coefficient between the USD score and EBL was $0.676(\mathrm{P}<0.001)$, indicating that there was a positive linear correlation between the score and EBL. A linear relationship between USD score and operative time was also observed ( $\mathrm{rs}=0.638, \mathrm{P}<0.001$ ), both of which indicated that the USD score was related to the difficulty and complexity of surgery.

Comparing the high and low surgical complexity groups of the test cohort, there was a significant difference between the USD aggregate score and divided item score, which consisted of the etiology score, segment score, and length score. In the validation group, a significant difference in the USD score was also found between the high and low complexity groups. The median USD score for high complexity surgeries was 7 points, and for low complexity surgeries was 4 points $(\mathrm{P}<0.001)$ (Figure 1A). A higher surgical complexity was correlated with a higher USD score (Figure 1B). Table 4 shows the distribution of UUTR surgery among USD classifications in the validation group.

Table 4 shows the variability of UUTR surgery in 
Table 2 Patient demographics and USD score and classification system of test group

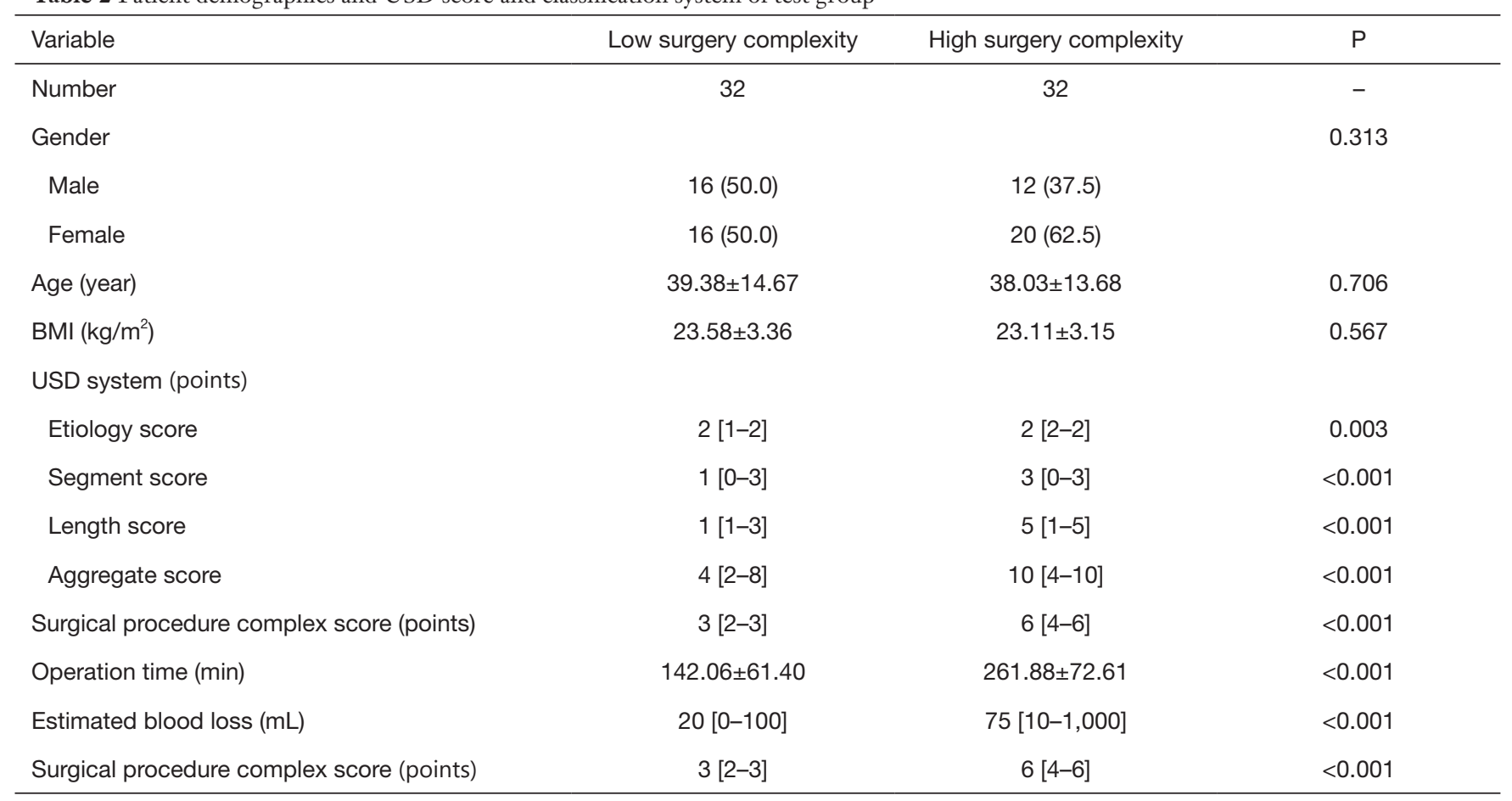

Data are presented in terms of mean \pm standard deviation, median [range] or $\mathrm{n}(\%)$. USD, ureter stricture disease; BMI, body mass index; EBL, estimated blood loss.

different USD classifications among 170 patients in the validation group, however with regularity to conform to. For E1S1L1 and E2S1L1 classifications, ureter stricture is located at the UPJ and the length of stenosis is less than or equal to $2 \mathrm{~cm}$ (L1). Thus, ureteropyeloplasty is the preferred option. Obviously, from the perspective of etiology, UUTR surgery of acquired causes (such as failure of previous surgery) is much more difficult than that of idiopathic ones. The same surgical procedure does not mean the same level of complexity. Through our USD classification system, surgical techniques could be compared reasonably under unified standard or background. According to the data analysis in our center, cystoscopy with ureteral dilation and stent placement is often applied to the middle or distal ureteral stenosis less than $2 \mathrm{~cm}$ (E1S0L1 E2S0L1 E2S1L1 E2S2L1), but it may take a risk of recurrence of stenosis. Onlay repair (buccal mucosae, lingual mucosae, appendix mucosae) is more frequently applied in the middle or proximal ureteral stenosis with 2-5 cm length (E2S2L1 E2S1L3 E2S2L1 E2S2L3). In the corresponding classifications, the onlay technique can avoid the disadvantages of high anastomotic tension in the end to end repair and reduce the intestinal complications caused by the ileal ureter replacement as well.

\section{Discussion}

USD is a relatively rare but underestimated problem for a variety of reasons. The choice of treatment is multifactorial and mainly depends on the extent of the defect and the time of diagnosis with respect to disease course $(4,7)$. The etiological mechanisms include ligation or suture kinking, partial or complete transection, clamp crushing, thermal injury, or ischemia due to devascularization (8). In many instances, endourological therapy with or without internal stent dilatation is the first choice for retrograde or anterograde intervention. The important role of stents in preventing urine extravasation, restructuring, and promoting ureteral healing must be weighed against the potential deterioration of the stricture severity reported by some authors (9). In case of contraindication or failure of endoscopic treatment, UUTR surgery after temporary urine diversion can be performed by open or minimally invasive techniques. All available surgical strategies are limited by the length and location of the ureter stricture itself but should comply with surgical principles, such as: spatulation 
Table 3 Patient demographics and USD score and classification system of validation group

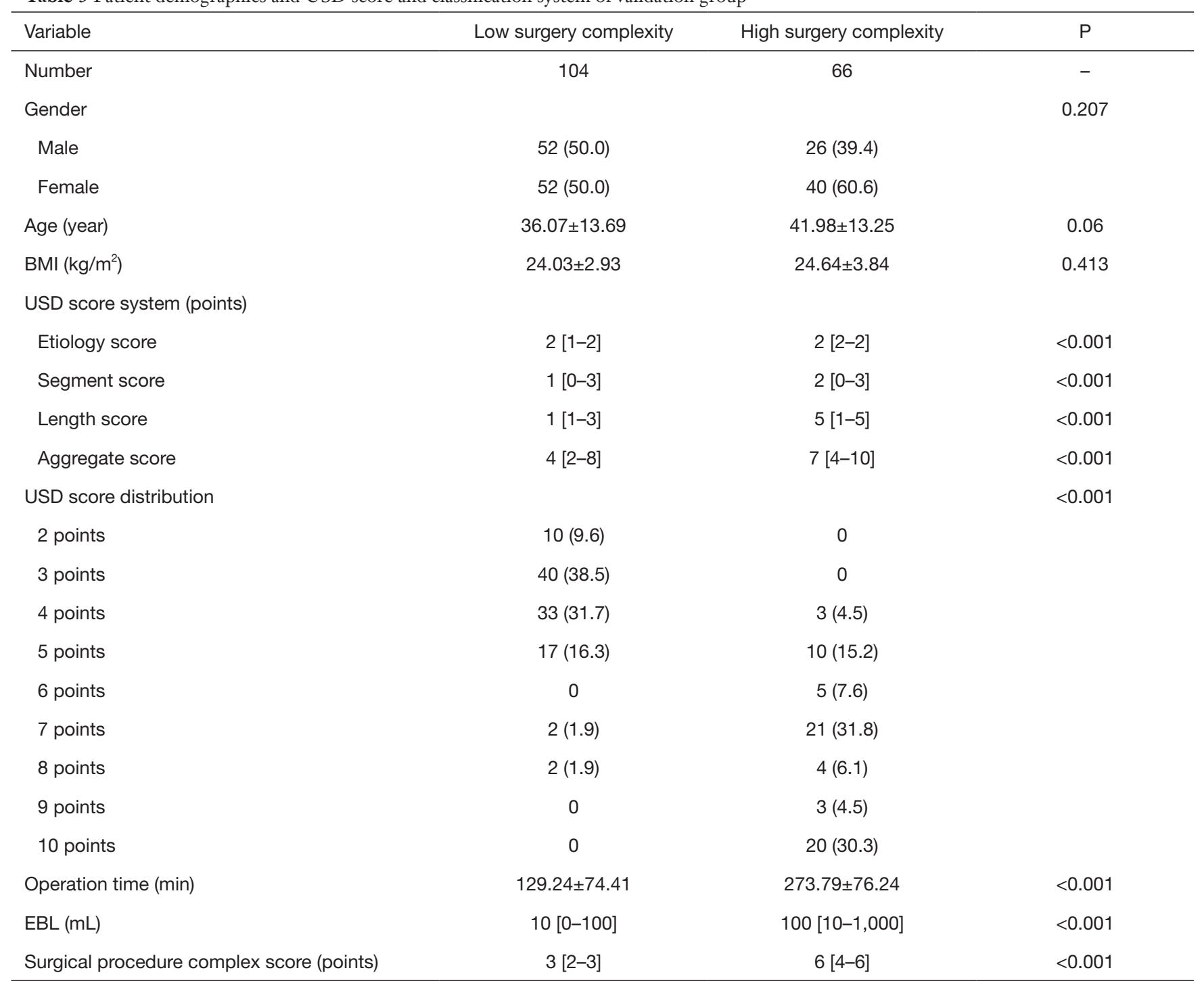

Data are presented in terms of mean \pm standard deviation, median [range] or $n(\%)$ USD, ureter stricture disease; BMI, body mass index; EBL, estimated blood loss.

of ureteral ends, mucosa-to-mucosa watertight anastomosis, external drainage, internal stenting, debridement of any necrotic tissue, and isolation with the peritoneum or omentum if possible $(10,11)$.

The treatment of stricture depends on a variety of parameters, the most important of which are its nature, segment, location, and severity. The choice of treatment is also based on preoperative decision-making between surgeons and patients. Under certain circumstances, the final decision of reparation is usually made in the operating room because it is there that the tissue tension and precise length of a stricture can be readily assessed $(7,11,12)$. Unfortunately, there are few standardized methods to gauge the stricture complexity or guide surgical decision-making.

To the best of our knowledge, the USD score and classification system comprise a novel system for quantifying ureter stricture characteristics and assessing the complexity of the UUTR surgical procedure. The system uses 3 stricture components: etiology (E), segment (S), and length (L). With the proposal and validation of the USD score and classification system, it is our goal to provide descriptive and quantitative methods for classifying USD complexity, and 

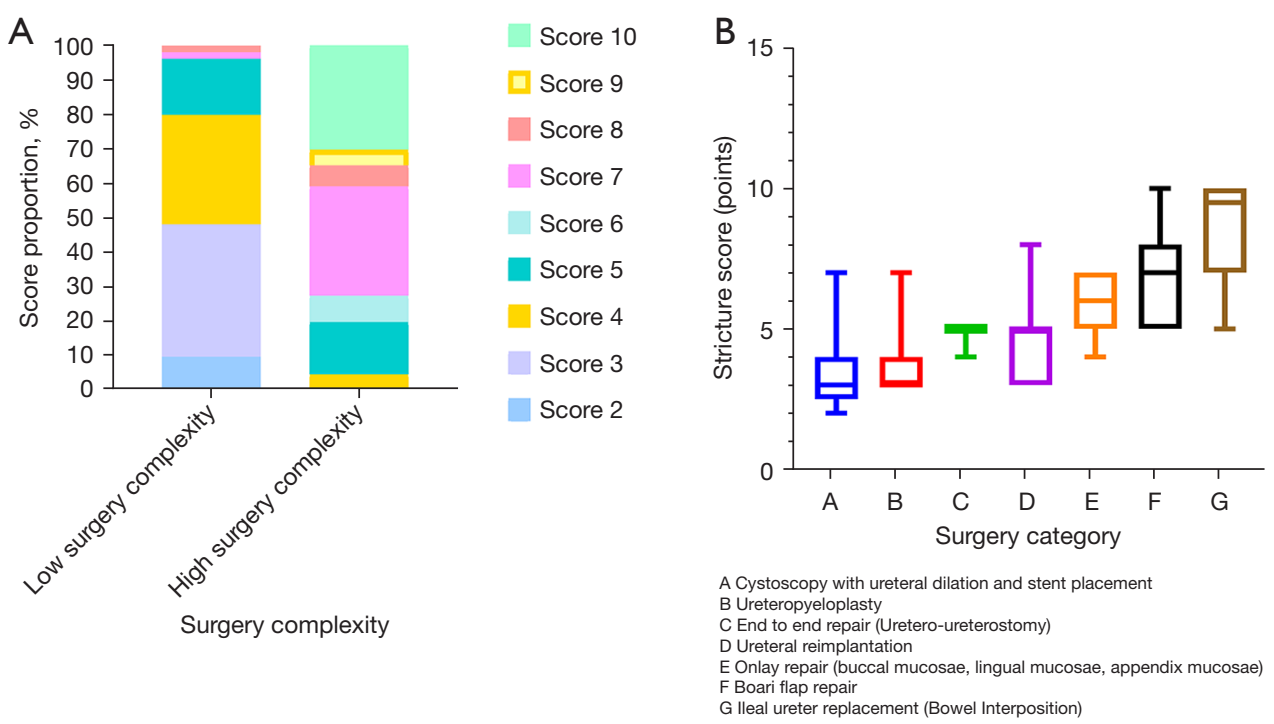

Figure 1 USD score and UUTR surgery. (A) USD score of low and high complexity of UUTR surgery, median USD score for high complexity surgeries was 7 points and for low complexity surgeries was 4 points $(\mathrm{P}<0.001)$. (B) USD score across the different UUTR surgery types, the median USD score for cystoscopy with ureteral dilation and stent placement, ureteropyeloplasty, end to end repair, ureteral reimplantation, onlay repair, Boari flap repair and Ileal ureter replacement was 3, 3, 5, 5, 6, 7 and 9.5, respectively. USD, ureteral stricture disease; UUTR, upper urinary tract reconstructive.

Table 4 Distribution of the UUTR surgery types across the different USD classifications

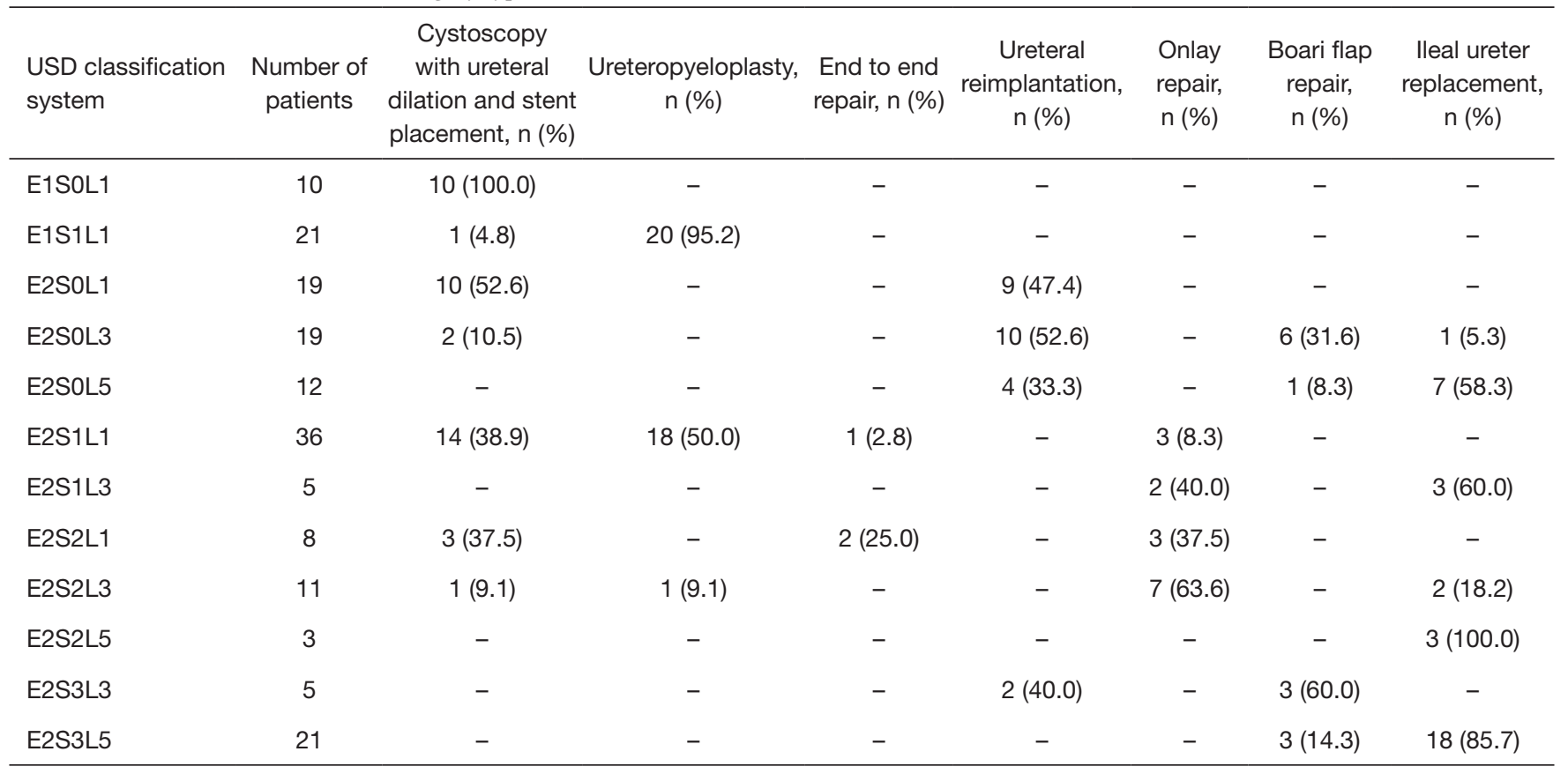

UUTR, upper urinary tract reconstructive; USD, ureter stricture disease; E score, etiology score; S score, segment score; L score, length score. 
objective methods for comparing various surgical techniques under a unified standard. When planning surgical strategies, the factors that should be taken into consideration include anatomical location and stricture length, as well as patient characteristics such as age, BMI, comorbidities, previous treatment, and even a preoperative assessment of bladder capacity (7). Because we were trying to develop a convenient and efficient descriptive system of scoring and classification, and our previous statistical analysis showed that the patient's age, gender, and BMI have little effect on the complexity of the operation, we did not include these variables because we did not want a system that was cumbersome and difficult to calculate. Prior to the development of the scoring and classification system, we conducted an extensive review of studies to identify candidate variables that are clinically useful and have been shown to independently influence surgical decisions $(6,13-15)$.

\section{Stricture etiology}

It is well known that the stricture etiology affects the complexity of UUTR surgery. Iatrogenic trauma and benign pathology are the most common causes of stricture (80\%) (16). Another $15 \%$ are of idiopathic origin, and the remainder are the result of malignant diseases. The incidence of ureter stricture varies by cause in urological surgical procedure, which can be seen in $1 \%$ of patients' post-ureteroscopy, 2-3\% post-pelvic radiation, 3-8\% post-renal transplantation, $1.4-15 \%$ in ureter-intestinal anastomoses, and $5-24 \%$ with stone impaction for more than two months $(4,17)$.

Non-ischemic or benign etiology is important while selecting patients most suitable for endoscopic management of USD, the endo-surgical management not associated with ischemic injury or radiotherapy is highly successful and results in minimal morbidity (18). UPJ obstruction may be either acquired or congenital, and can be due to extrinsic or intrinsic forces. The most common cause of UPJ obstruction is a congenital abnormality due to an anterior vessel crossing to the lower pole, an aperistaltic segment of the ureter, ureteral kinking, or a high insertion (19). Minimally invasive ureteropyeloplasty has emerged as the gold standard for UPJ obstruction reparation with low postoperative morbidity and high success rates (20).

\section{Segment and length}

Probably weighted as the most important factor in the ureter stricture is the segment and length of the stricture. The ureter stricture segment is defined as proximal (UPJ to the sacroiliac joint), middle (overlying the sacroiliac joint), or distal (sacroiliac joint to the ureterovesical junction) (21). Combined with urography (antegrade and retrograde) and diuretic renal scan, the presence of obstruction can be confirmed, and the stricture segment and length can be accurately evaluated. If a pyelogram cannot be successfully performed, then the ideal imaging technique is a computed tomography (CT) urography with a 3-dimensional reconstructive technique or cine MRU for optimal anatomy visualization $(22,23)$.

Proximal ureter stricture is the rarest site, and is mainly caused by iatrogenic injury (22). Short ureteral transections or strictures of the middle ureter $(1-2 \mathrm{~cm})$ can be repaired by end-to-end anastomosis (ureteroureterostomy) (24). In the cases of long-distance middle ureter stricture, ureteroureterostomy cannot be performed. Here, the ureter can (I) be displaced through the midline and anastomosed with the contralateral ureter, (II) be substituted by an ileal segment, (III) be shunted from the renal pelvis to the bladder, (IV) be treated with ureterolysis and subsequent omentum majus wrapping, or (V) kidney autologous transplantation is performed into the small pelvis. Another option is to place a permanent ureteral stent. For the management of long proximal/middle ureteral strictures (2-6 cm), Cheng et al. reported that minimally invasive onlay flap/graft (appendiceal flap, lingual mucosa graft, and buccal mucosa graft) ureteroplasty was proven to be safe and feasible (6). A short distal ureter stricture is usually treated by removing the injured ureteral segment followed by ureter reimplantation. Using the Psoas-Hitch/Boari-flap technique, a larger distance can be covered, so as to achieve tension-free anastomosis. The Psoas-Hitch technique can be used when there is $3-4 \mathrm{~cm}$ stricture length and normal bladder capacity, and the Boari-flap technique is used for ureter stricture of up to $7-8 \mathrm{~cm}(25,26)$.

As the results showed, a linear relationship between USD score and operative time or EBL was also observed. Based on the total points from etiology, segment, and length in USD, the score can assist surgeons in predicting the operative complexity. The proposed classification system will aid in categorizing ureter strictures, surgical planning, and clinical communications (Figure 2). For example, the description of "this patient has a $1 \mathrm{~cm}$, UPJO due to congenital extrinsic forces" could be standardized as "E1S1L1 3 points", which would be managed by ureteropyeloplasty (Figure $2 A, 2 B$ ), or the statement "this 


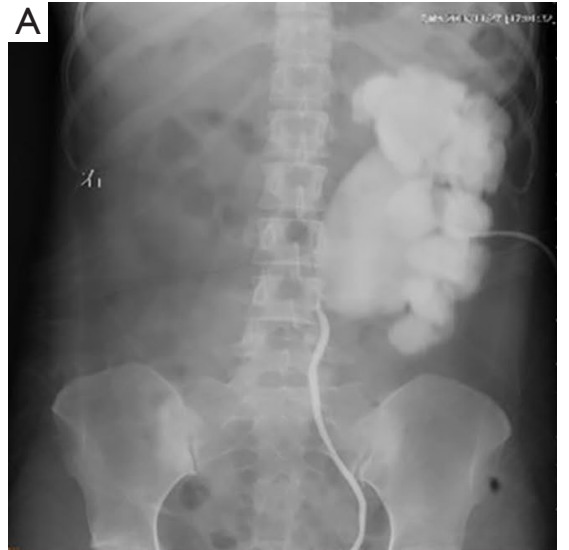

B

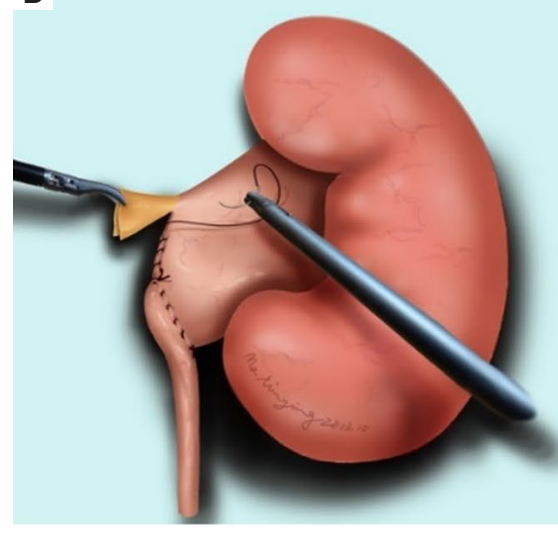

C

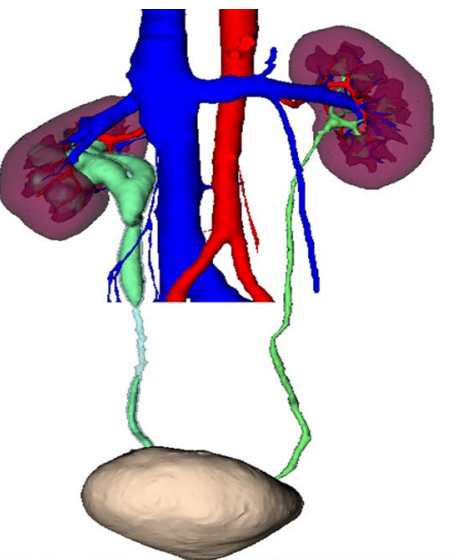

D

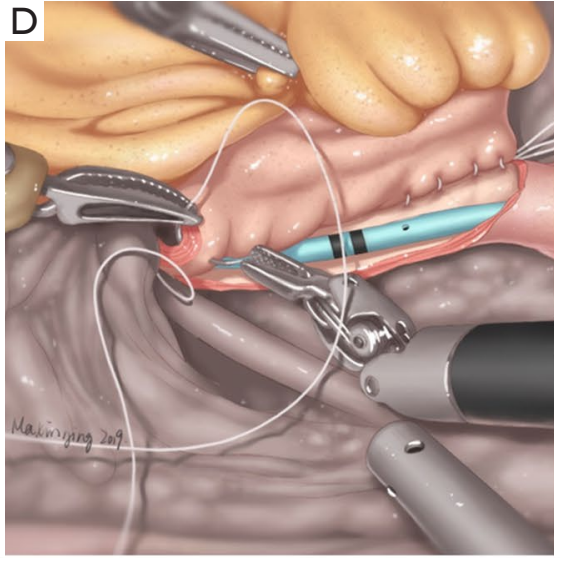

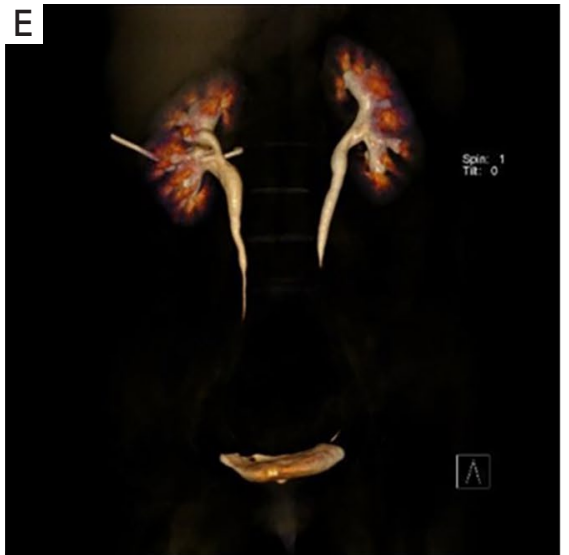

F

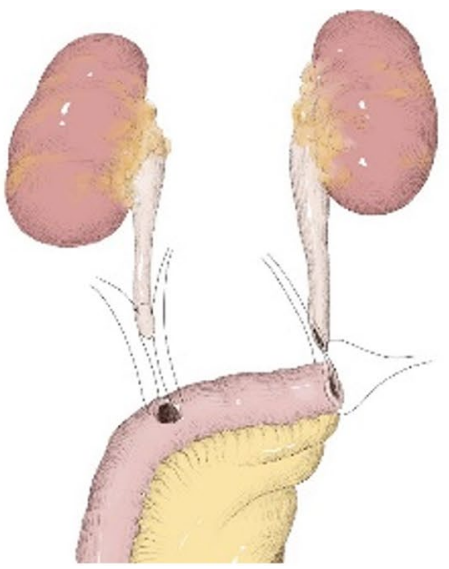

Figure 2 USD classification system aiding in categorizing ureter strictures and clinical strategy. (A,B) The description of "One 1 cm, UPJO due to congenital extrinsic forces" could be standardized as "E1S1L1 3points", which was managed by laparoscopic dismembered ureteropyeloplasty. (B) was originally published in Yang K, Yao L, Li X, et al. (27). Re-use permission was obtained. (C,D) The description of "One $5 \mathrm{~cm}$, middle ureter stricture caused by iatrogenic trauma" could be standardized as "E2S2L5 9points", which was managed by appendiceal onlay flap ureteroplasty. (E,F) The description of "Long ureter strictures on both side secondary to pelvic radiotherapy" could be standardized as "E2S3L5 10points", which was managed by laparoscopic bilateral ileal ureter substitution. USD, ureteral stricture disease.

patient has a $5-\mathrm{cm}$ middle ureter stricture caused by iatrogenic trauma" is classified as "E2S2L5 9 points" which would be managed by onlay flap/graft ureteroplasty or even ileal ureter replacement (Figure 2C,2D). For "long ureter strictures on both sides secondary to pelvic radiotherapy", we classified it as "E2S3L5 10 points," which would be solved by bilateral ileal ureter substitution (Figure 2E,2F).

In general, the USD classification system can assist in preoperative planning. Especially for young urologists, the classification can assist with predicting the complexity of surgery. Through the USD classification system, patients can acquire a more detailed understanding of their conditions, and physicians can provide patients with operative alternative options. Ultimately, doctors and patients can negotiate and choose the most optimal surgical methods. However, Table 4 reflects the choice and preference of our center's urologists for UUTR surgery. As far as E2S0L3 is concerned, there are 4 types of options from which urologists may choose. Therefore, choosing the most optimal UUTR plan is not simple, which is based on the USD classification system, and other factors should also be taken into consideration. We will further expand the sample size, promote multi-center cooperation, and comprehensively and systematically conduct further research on the correlation between the USD classification system and the surgical plan to eliminate the bias. 
The USD score and classification system was originally developed to be similar to the R.E.N.A.L. Nephrometry Score, to predict the complexity of surgery, and assist in preoperative decision-making $(28,29)$. The current study proved that the system based on 3 factors easily obtained from the patient history, plus a physical exam and preoperative radiological images, had a sufficiently high interobserver reliability that can be used in clinical practice by urologists. Then, we validated that this system correlates to UUTR surgical complexity. A higher USD score was correlated with a higher surgical complexity. Therefore, it can be used for assessment of the surgical complexity and comparison of USD treatment under a unified standard. Additionally, once the stricture was categorized, it would assist in predicting the type of surgery for surgeons in clinical practice. We were able to successfully confirm the validity of the USD score and classification system to provide a quantitative and descriptive means of classifying ureter stricture complexity, and to predict the complexity of UUTR surgery.

Our study had several limitations. First, the shortcomings of the study include its relatively small number of patients and the retrospective nature of the UUTR surgical procedure in the test and validation groups. Standardizing and validating the ureter stricture should allow for collaboration among institutions in the future, which will be able to create a widely accepted "standard", provide a higher level of evidence for determining the optimal treatment and therefore improve the quality of USD research. Second, unlike R.E.N.A.L. Nephrometry Score which could be independently associated with the occurrence of complications and predict malignant and high-grade pathology. We did not explore the relationship between the USD score and classification system and clinical outcome in the current study. To obtain additional data, future studies correlating the system with outcomes such as anastomosis leak and stricture recurrence will be carried out. In addition, there will theoretically be $24+$ different types of strictures ( 2 etiologies $\times 4$ segments $\times 3$ lengths). Obviously, many of these combinations will be rare and of little clinical significance, but it is our goal to finally determine that each type of stricture does have a most optimal management method. The validated USD score and classification system will be used as a tool to be approached with an algorithmic model that determines the decision for UUTR technique by etiology, segment, and length.

\section{Conclusions}

The USD score and classification system we propose herein is a reliable, effective, and validated grading scale for describing the surgical complexity of UUTR surgery. The three objective factors that comprise the system are vital for determining the most optimal reconstructive procedure. Urologists can improve the quality of study for USD by using the universally understood and applicable method of describing the ureter stricture. Research with a large sample is needed to further examine and modify the use of the system.

\section{Acknowledgments}

The authors thank the entire staff of the Department of Urology, Peking University First Hospital.

Funding: None.

\section{Footnote}

Reporting Checklist: The authors have completed the STROBE reporting checklist. Available at https://dx.doi. org/10.21037/tau-21-575

Data Sharing Statement: Available at https://dx.doi. org/10.21037/tau-21-575

Conflicts of Interest: All authors have completed the ICMJE uniform disclosure form (available at https://dx.doi. org/10.21037/tau-21-575). XL and LZ serve as unpaid editorial board members of Translational Andrology and Urology. The other authors have no conflicts of interest to declare.

Ethical Statement: The authors are accountable for all aspects of the work in ensuring that questions related to the accuracy or integrity of any part of the work are appropriately investigated and resolved. All procedures performed in this study were in accordance with the Declaration of Helsinki (as revised in 2013) and approved by the Ethics Committee of Peking University First Hospital (approval number: 2020-283). Because of the retrospective nature of the research, the requirement for informed consent was waived.

Open Access Statement: This is an Open Access article 
distributed in accordance with the Creative Commons Attribution-NonCommercial-NoDerivs 4.0 International License (CC BY-NC-ND 4.0), which permits the noncommercial replication and distribution of the article with the strict proviso that no changes or edits are made and the original work is properly cited (including links to both the formal publication through the relevant DOI and the license). See: https://creativecommons.org/licenses/by-nc-nd/4.0/.

\section{References}

1. Dahlkamp L, Haeuser L, Winnekendonk G, et al. Interdisciplinary Comparison of PADUA and R.E.N.A.L. Scoring Systems for Prediction of Conversion to Nephrectomy in Patients with Renal Mass Scheduled for Nephron Sparing Surgery. J Urol 2019;202:890-8.

2. Brockman JA, Alanee S, Vickers AJ, et al. Nomogram Predicting Prostate Cancer-specific Mortality for Men with Biochemical Recurrence After Radical Prostatectomy. Eur Urol 2015;67:1160-7.

3. Alwaal A, Sanford TH, Harris CR, et al. Urethral Stricture Score is Associated with Anterior Urethroplasty Complexity and Outcome. J Urol 2016;195:1817-21.

4. Tyritzis SI, Wiklund NP. Ureteral strictures revisited... trying to see the light at the end of the tunnel: a comprehensive review. J Endourol 2015;29:124-36.

5. Buffi NM, Lughezzani G, Hurle R, et al. Robot-assisted Surgery for Benign Ureteral Strictures: Experience and Outcomes from Four Tertiary Care Institutions. Eur Urol 2017;71:945-51.

6. Cheng S, Fan S, Wang J, et al. Laparoscopic and robotic ureteroplasty using onlay flap or graft for the management of long proximal or middle ureteral strictures: our experience and strategy. Int Urol Nephrol 2021;53:479-88.

7. Paffenholz P, Heidenreich A. Modern surgical strategies in the management of complex ureteral strictures. Curr Opin Urol 2021;31:170-6.

8. Delacroix SE Jr, Winters JC. Urinary tract injures: recognition and management. Clin Colon Rectal Surg 2010;23:104-12.

9. Yossepowitch O, Lifshitz DA, Dekel Y, et al. Predicting the success of retrograde stenting for managing ureteral obstruction. J Urol 2001;166:1746-9.

10. Png JC, Chapple CR. Principles of ureteric reconstruction. Curr Opin Urol 2000;10:207-12.

11. Kapogiannis F, Spartalis E, Fasoulakis K, et al. Laparoscopic and Robotic Management of Ureteral Stricture in Adults. In Vivo 2020;34:965-72.
12. Reus C, Brehmer M. Minimally invasive management of ureteral strictures: a 5-year retrospective study. World J Urol 2019;37:1733-8.

13. Zhu W, Xiong S, Fang D, et al. Minimally invasive ileal ureter replacement: Comparative analysis of robot-assisted laparoscopic versus conventional laparoscopic surgery. Int J Med Robot 2021;17:e2230.

14. Ding G, Cheng S, Li X, et al. Experience managing distal ureteral strictures with Boari flap-psoas hitch and comparison of open and laparoscopic procedures. Transl Androl Urol 2021;10:56-65.

15. Cheng S, Li X, Yang K, et al. Modified Laparoscopic and Robotic Flap Pyeloplasty for Recurrent Ureteropelvic Junction Obstruction with a Long Proximal Ureteral Stricture: The "Wishbone" Anastomosis and the "Ureteral Plate" Technique. Urol Int 2021;105:642-9.

16. Elliott SP, McAninch JW. Ureteral injuries: external and iatrogenic. Urol Clin North Am 2006;33:55-66, vi.

17. Roberts WW, Cadeddu JA, Micali S, et al. Ureteral stricture formation after removal of impacted calculi. J Urol 1998;159:723-6.

18. Kachrilas S, Bourdoumis A, Karaolides T, et al. Current status of minimally invasive endoscopic management of ureteric strictures. Ther Adv Urol 2013;5:354-65.

19. Borin JF. Ureteropelvic Junction Obstruction in Adults. Rev Urol 2017;19:261-4.

20. Nishi M, Matsumoto K, Fujita T, et al. Improvement in Renal Function and Symptoms of Patients Treated with Laparoscopic Pyeloplasty for Ureteropelvic Junction Obstruction with Less Than 20\% Split Renal Function. J Endourol 2016;30:1214-8.

21. Simmons MN, Gill IS, Fergany AF, et al. Laparoscopic ureteral reconstruction for benign stricture disease. Urology 2007;69:280-4.

22. Engel O, Rink M, Fisch M. Management of iatrogenic ureteral injury and techniques for ureteral reconstruction. Curr Opin Urol 2015;25:331-5.

23. Zhu WJ, Ma MM, Zheng MM, et al. Cine magnetic resonance urography for postoperative evaluation of reconstructive urinary tract after ileal ureter substitution: initial experience. Clin Radiol 2020;75:480.e1-480.e9.

24. Thiel DD, Badger WJ, Winfield HN. Robot-assisted laparoscopic excision and ureteroureterostomy for congenital midureteral stricture. J Endourol 2008;22:2667-9.

25. McClung C, Gorbonos A. Ureteral reimplantation in adults: open versus robotic. J Urol 2014;192:1023-5.

26. Do M, Kallidonis P, Qazi H, et al. Robot-assisted 
technique for boari flap ureteral reimplantation: is robot assistance beneficial? J Endourol 2014;28:679-85.

27. Yang K, Yao L, Li X, et al. A modified suture technique for transperitoneal laparoscopic dismembered pyeloplasty of pelviureteric junction obstruction. Urology 2015;85:263-7. 28. Hayn MH, Schwaab T, Underwood W, et al. RENAL

Cite this article as: Zhu W, Zhu Z, Li Z, Li X, Zhang J, Xu Y, Wang X, Zhang P, Huang B, Huang C, Zhang D, Zhu H, Li X, Lin J, Zhou L. A ureteral stricture disease score and classification system: correlation with upper urinary tract reconstructive surgery complexity. Transl Androl Urol 2021;10(10):3745-3755. doi: 10.21037/tau-21-575 nephrometry score predicts surgical outcomes of laparoscopic partial nephrectomy. BJU Int 2011;108:876-81.

29. Kutikov A, Uzzo RG. The R.E.N.A.L. nephrometry score: a comprehensive standardized system for quantitating renal tumor size, location and depth. J Urol 2009;182:844-53. 